\title{
RESGATE HISTÓRICO E CIDADANIA: MANUTENÇÃO DO PATRIMÔNIO MATERIAL E IMATERIAL DA ESCOLA DE ENFERMAGEM DE RIBEIRÃO PRETO - USP
}

\author{
HISTORICAL RESCUE AND SOCIAL RESPONSIBILITY: MAINTENANCE OF \\ TANGIBLE AND INTANGIBLE HERITAGE OF UNIVERSITY OF SÃO PAULO \\ AT RIBEIRÃO PRETO COLLEGE OF NURSING
}
*Luciana Barizon Luchesi, **Mara Elisa Ferreira Oliva, ***Toyoko Saeki, ****Margarita Antonia Villar Luis, ******Isabel Amélia Costa Mendes

\section{RESUMO}

Descreve-se, aqui, a história e funcionamento do Centro de Memória da Escola de Enfermagem de Ribeirão Preto no âmbito do projeto Resgate Histórico e Cidadania: Manutenção do Patrimônio Material e Imaterial da Escola de Enfermagem de Ribeirão Preto - USP, traçando os determinantes históricos que o colocam como centro de rico potencial para a pesquisa em diversas áreas, não apenas no campo da História da Enfermagem, mas, também, Moda, Gênero, entre outros. Apresentam-se as estratégias e parcerias desse projeto, que busca não apenas a conservação preventiva de seu patrimônio, mas também o despertar de vocações para a pesquisa em História da Enfermagem e o incentivo à produção de pesquisadores através de auxílio na busca de fontes pertinentes.

Palavras-chave: História da Enfermagem. Acervo de biblioteca. Museu.

\section{ABSTRACT}

It describes the history and work on the Memory Center Museum of University od São Paulo College of Nursing at Ribeirão Preto, in the context of the project Historical Rescue and Social Responsibility: Maintenance of Tangible and Intangible Heritage of University of São Paulo at Ribeirão Preto College of Nursing, tracing the historical determinants that place as a rich potential center for research in several areas, not only in the field of History of Nursing, but also Fashion, Gender, among others. Presents the strategies and partnerships that this project that seeks not only preventive conservation of its heritage, but also the awakening of talents to the History of Nursing research and encourage the studies of researchers, through assistance in finding relevant sources.

Key words: History of Nursing. Library materials. Museum.

\footnotetext{
* Enfermeira, professora doutora do Departamento de Enfermagem Psiquiátrica e Ciências Humanas da Escola de Enfermagem de Ribeirão Preto (EERP-USP), diretora de Assuntos Científico-Culturais da Academia Brasileira de História da Enfermagem (ABRADHENF), líder do Laboratório de Estudos em História da Enfermagem (LAESHE), integrante do Laboratório de Abordagens Científicas na História da Enfermagem (LACENF). Escola de Enfermagem de Ribeirão Preto - Avenida Bandeirantes, 3900 I4048-900 - Ribeirão Preto - SP - e-mail: luchesi@eerp.usp.br. ** Pedagoga, especialista em História, responsável pelo Centro de Memória da EERP-USP - e-mail: mara@eerp.usp.br. *** Enfermeira, professora doutora do Departamento de Enfermagem Psiquiátrica e Ciências Humanas da EERP-USP - e-mail: maryto@eerp.usp.br. ***** Enfermeira, professora titular do Departamento de Enfermagem Psiquiátrica e Giências Humanas da EERP-USP, membro do LAESHE - e-mail: margarit@eerp.usp.br. ******Enfermeira, professora titular do Departamento de Enfermagem Geral e Especializada da EERP-USP - e-mail: iamendes@usp.br.
} 


\section{INTRODUÇÃO}

\section{BREVE HISTÓRICO DA ESCOLA DE ENFERMAGEM DE RIBEIRÃO PRETO}

As atividades desenvolvidas através do Projeto Resgate Histórico e Cidadania: Manutenção do Patrimônio Material e Imaterial da Escola de Enfermagem de Ribeirão Preto - USP não são pioneiras. Trata-se da continuidade de projetos iniciados em 2003, com o objetivo de preservar o patrimônio material e imaterial da EERP-USP.

A importância desse arquivo para a História da Enfermagem apenas pode ser compreendida observando-se a trajetória da instituição e do próprio arquivo histórico.

A EERP-USP foi criada em 26 de dezembro de I95I pela Lei Estadual n ${ }^{0}$ I.467 [3]. Contudo, as aulas tiveram início em IO de agosto de I953. Pode-se dizer, até o momento atual, que a história da EERP não deve ser contada sem a história de sua primeira diretora, a professora Glete de Alcântara. Para isso, é necessário voltar um pouco mais no tempo.

A professora Glete de Alcântara, nascida aos 24 de junho de I9IO, realizou curso de educadora sanitária pelo Instituto de Higiene de São Paulo em I930 [I]. Fato que possibilitou, através de contato com a educadora sanitária Maria Rosa de Sousa Pinheiro, sua diplomação em Enfermagem pela Universidade de Toronto, Canadá, como bolsista da Fundação Rockefeller, em um acordo que tinha como um de seus objetivos a implementação e estruturação da Escola de Enfermagem da USP (EE-USP) [9].

As bolsas concedidas nesse convênio tinham como demanda que, retornando ao Brasil, as bolsistas seriam contratadas como docentes da EE-USP. Em seu retorno, em I944, após a diplomação, a professora Glete de Alcântara confirmou as exigências referidas, passando a lecionar Técnica de Enfermagem e Enfermagem Médica na EE-USP [I, 9].

Em I947, a professora inicia o curso de licenciatura em Ciências Sociais, na Faculdade de Filosofia, Ciências e Letras da Universidade de São Paulo, curso interrompido em I950 por um ano, quando vai a estudos ao Teachers College, na Universidade de Columbia, EUA, onde obteve o título de Master of Arts. Ao retornar, finaliza o curso de licenciatura, graduando-se em I952 [I].

Ainda na década de I940, um grande movimento na USP inicia as discussões sobre a necessidade de uma nova Faculdade de Medicina e que essa deveria ser implementada no interior paulista, visto a alta procura pelo curso e dificuldades de absorção de estudantes com boas notas no vestibular. A Comissão de Ensino e Regimento da Universidade de São Paulo, comissão responsável por estudar a viabilidade da proposta, presidida por Prof. Dr. Zeferino Vaz, conclui pela viabilidade da mesma, decidindo a USP, posteriormente, por sua implementação na cidade de Ribeirão Preto [8].

A mesma comissão relatora destaca a importância da anexação de uma escola de Enfermagem à nova escola de Medicina, pois isso seria primordial para o funcionamento do Hospital das Clínicas e para o desenvolvimento da saúde na região, onde a escola de Enfermagem seria instalada ${ }^{\dagger}[\mathrm{II}]$.

Com a criação da Faculdade de Medicina de Ribeirão Preto, em I948 - e sua efetiva estruturação, em dezembro de I95I, documento que traz em seu bojo, no art. I3, a criação da EERP-USP, anexa -, foi convidada a professora Glete de Alcântara para a direção daquela escola de Enfermagem, que terá destaque em nível nacional e internacional [3]. Desde I947, foi galgando espaço dentro da Associação Brasileira de Enfermagem, chegando à diretora dessa importante associação nacional por duas ocasiões [I, I5].

Outra de suas colaborações foi o pioneirismo na pós-graduação, ao defender, em I963, a primeira tese de cátedra de um enfermeiro latino-americano, A enfermagem moderna como categoria profissional: obstáculos à sua expansão na sociedade brasileira, fato que registrou seu nome nas páginas da História da Enfermagem Brasileira [2].

Muito dessa história pode ser analisada através de um rico acervo histórico, mas a falta de funcionários especializados deixou o arquivo, de I99I-I999, pouco utilizado e com organização em papel, o que dificultava o acesso rápido às informações.

\section{UM ARQUIVO PIONEIRO?}

A criação do Centro de Memória da Escola de Enfermagem de Ribeirão Preto - USP (Cemeerp) deu-se em I988, durante a comemoração dos 35 anos da instituição [I3]. Em maio do ano seguinte, a Portaria D/EERP-I8/89 estabelece uma comissão responsável por esse centro, composta por Nadyr Viana Lomônaco (coordenadora), Profa. Dra.' Daisy Leslie Steagall Gomes, prof. Francisco de Assis Correia e a sra. Maria José Cesarino Fram.

$\dagger$ UNIVERSIDADE DE SÃO PAULO, Processo ${ }^{\circ}{ }^{\circ} 3 \cdot 320$, referente ao relatório apresentado pela Comissão de Ensino e Regimento da Universidade de São Paulo ao Conselho Universitário da Universidade de São Paulo, sobre a instalação da Faculdade de Medicina de Ribeirão Preto, I95I. 
A referida comissão realizou uma série de treinamentos junto ao Instituto de Estudos Brasileiros da USP, o Arquivo Público e Histórico do Município de Rio Claro, em SP, e o Centro de Memória da Unicamp, cujo docente, prof. Fernando Antônio Abrahão, que também visitou o acervo, ajudou na elaboração dos objetivos do mesmo [2I].

O grupo, inicialmente, empenhou-se em reunir objetos e construir fontes orais, acerca dos primórdios da EERP-USP, conservando adequadamente os registros do processo de sua criação e estruturação. Para o arquivo de fontes orais, o grupo contou com assessoria do prof. Carlos Humberto P. Corrêa, da Universidade Federal de Santa Catarina. Ao término dos trabalhos, houve a inauguração do espaço em 5 de março de I990 [13]. Entretanto, dois meses depois, a Portaria D/EERP-27/90 extinguiu a Portaria que estabelecia a comissão para atuação na Memória Histórica.

Em I99I, a então diretora, a Profa. Dra. Vera Heloísa Pileggi Vinha, emitiu a Portaria D/EERP 49/9o, em I4 de agosto, estabelecendo novamente a Comissão de Memória Histórica, cujo objetivo principal era dar continuidade à organização do Cemeerp. A comissão contava com o professor Francisco de Assis Correia (presidente), a Profa. Dra. Daisy Leslie Steagall Gomes, os professores Zaíra Benedini, Nadyr Viana Lomônaco, Francisco José Barroso Vessi, Maria José Cesarino Fram, a aluna de pós-graduação Eliana Faria de Angelice Biffi e as alunas de graduação Marisa Júlio Ragoso e Andréia Pádua Pereira.

O processo de criação do Centro de Memória não difere do ocorrido em outras importantes instituições. Aparentemente, o jubileu de uma instituição instiga nos profissionais busca pela identidade institucional e pelos avanços, que não podem ser entendidos sem a compreensão histórica de cada momento, fato que demanda debruçar-se sobre as fontes documentais e criar novas fontes: orais, iconográficas, audiovisuais, entre outros.

Para surpresa de muitas instituições, esse é o momento do balanço da história perdida, pois anos ou décadas sem uma prática de conservação ou catalogação adequada levaram muito da história documental para o lixo por desconhecimento, ou por excesso de infestações, causados por mau acondicionamento.

Para Sauthier [I9], a prática de "desobstrução" de espaços liquidou com muito da memória profissional da Enfermagem e ainda se carece de diagnóstico geral dos arquivos de Enfermagem no país. No ano de
2000, a autora já chamava a atenção para a necessidade de políticas nacionais de preservação para os acervos históricos da Enfermagem.

Dessa forma, ao realizar busca por literatura a respeito da criação de acervos para a História da Enfermagem, vê-se que alguns deles, dos mais importantes do país, têm data de criação após o ano de I990. Poder-se-ia, então, pensar no título de arquivo pioneiro da Enfermagem no Brasil para o Cemeerp? Depende do referencial que se toma para essa medida. Se for a data de criação oficial, para arquivos dentro de instituições de Enfermagem, parece que a resposta seria sim. Caso contar-se os esforços para manutenção de fontes e preocupação com a história da instituição, talvez a resposta seja negativa.

O Centro Histórico-Cultural da Enfermagem Ibero-Americana foi fundado em 20 de outubro I992, nas comemorações do jubileu de ouro da Escola de Enfermagem da USP, localizado na capital paulista. A ampliação do nome deveu-se a convênios internacionais, em vigência na época, que facilitariam o intercâmbio de pesquisadores [I7].

Apesar de o Centro de Documentação da Escola de Enfermagem Anna Nery ter sido inaugurado em 8 de dezembro de I993, comemorando a criação do Centro, a primeira tese da instituição, na linha de pesquisa histórica, e a criação do Núcleo de Pesquisa de História da Enfermagem Brasileira (Nuphebras), a organização do acervo para fins de pesquisa estava em andamento desde a década de I960 [I9]. O referido núcleo de pesquisa imprimiu ritmo acelerado na pesquisa em História da Enfermagem na sua primeira década de existência, promovendo pesquisas importantes em nível de pós-graduação stricto sensu e eventos internacionais importantes, como o Colóquio Latino-americano de História da Enfermagem, nos anos 2000 e 2005.

Para Barreira e Baptista [6], a Casa de Oswaldo Cruz, criada em I986 no Rio de Janeiro, apesar de não estar alocada em uma escola de Enfermagem, também se trata de rico acervo para a História da Enfermagem Brasileira. Possui documentos sobre os primórdios da Escola de Enfermagem Anna Nery, sobre a Fundação Rockefeller e o antigo Serviço Especial de Saúde Pública (Sesp).

O Centro de Memória Dra. Nalva Pereira Caldas, da Faculdade de Enfermagem da Universidade do Estado do Rio de Janeiro - UERJ foi criado em I8 de junho de I998, nas comemorações do jubileu de ouro da faculdade. Entretanto, um dos subprojetos que deram origem à sua organização é de I995 [7]. 
Em 2005, a Escola de Enfermagem Alfredo Pinto (EEAP - Unirio) inicia a implantação do Arquivo Setorial Enfermeira Maria de Castro Pamphiro que, apesar de sua organização recente, possui fontes que datam de I89o a 2005 , constituindo um dos acervos mais importantes do país para a pesquisa em História da Enfermagem. O Laboratório de Pesquisa em História da Enfermagem (LAPHE), da EEAP, criado em 2000, já possui ampla visibilidade no cenário da pesquisa nacional [20].

Em pleno funcionamento também está o Centro de Memória da Escola de Enfermagem da Universidade Federal de Minas Gerais, desde sua criação, em 22 de fevereiro de 2006. Entretanto, os responsáveis afirmam que sua organização teve início em I994 [IO].

O Instituto do Patrimônio Histórico e Artístico Nacional (IPHAN), brasileiro, tem nomenclaturas para dois tipos de patrimônio: imaterial e material. O patrimônio imaterial refere-se a "práticas, representações, expressões, conhecimentos e técnicas, instrumentos, objetos, artefatos e lugares, são reconhecidos por comunidades como parte integrante de seu patrimônio cultural". Sua transmissão é feita de uma geração para outra e adaptado para cada ambiente e contexto histórico [4]. Nesse sentido, pode-se referir como exemplos de patrimônio imaterial as cerimônias de formatura na Enfermagem, o rito de passagem da lâmpada, o uniforme e touca da enfermeira, e uma série de outros ritos, próprios da Enfermagem brasileira.

Para o IPHAN, o patrimônio material é classificado segundo a natureza dos bens culturais. Podem ser divididos em bens imóveis e móveis, onde se incluem "acervos museológicos, documentais, bibliográficos, arquivísticos, videográficos, fotográficos e cinematográficos" [4].

Rico conjunto de fontes que representam o patrimônio material e imaterial da EERP-USP foi organizado e catalogado em papel e fazem parte do acervo: Portarias; Decretos que tratam da documentação administrativa da Escola de Enfermagem; arquivo iconográfico estimado em mais de I5 mil itens; negativos de fotos de várias épocas da EERP-USP; arquivos de alunos; arquivos de eventos; fontes orais em suporte de fita cassete, com registro oral de funcionários, alunos e professores; arquivos de fitas de vídeo VHS, com informações sobre eventos científicos e culturais; arquivos de CDs e discos de vinil; arquivos de condecorações, como troféus, placas, medalhas de menções honrosas e souvenirs. Possui, também, mobiliários, vestimentas, quadros e diversas peças de instrumental cirúrgicos, entre muitos outros. Entretanto, apesar dos muitos esforços da instituição, o Cemeerp manteve-se pouco ativo até I999.

Em I999, o Grupo de Estudo e Pesquisas em Comunicação no Processo em Enfermagem (GEPEGOPEn), liderado pela Profa. Dra. Isabel Amélia Costa Mendes, organizou o fórum Memória e história institucional, que buscou sensibilizar todos os setores da EERP-USP para a preservação e organização documental da instituição. No mesmo ano, houve a aprovação de uma Bolsa Trabalho por um ano, o que colaborou para a abertura do arquivo ao público, mas que não foi renovada [I4]

Em 2002, um trabalho voluntário, da então enfermeira Luciana Barizon Luchesi (pesquisadora na área de História da Enfermagem) e de uma aluna de graduação, Lia Mara da Silva, com assessoria de docentes e amplo apoio da direção da EERP-USP, iniciou processo de apoio permanente do acervo, com o objetivo de preservá-lo, auxiliar na busca de fontes para os projetos da comunidade científica e ampliação do acesso público, despertando vocações para a pesquisa em História da Enfermagem e contribuindo para a formação acadêmica, ensino, pesquisa e extensão [I4].

\section{ESTRATÉGIAS PARA OBTENÇÃO DE FOMENTO: HISTÓRICO DOS PROJETOS DE EXTENSÃO DO GRUPO}

Algumas estratégias foram realizadas no sentido de promover a manutenção dos trabalhos, visto que a organização e o acondicionamento adequado dos documentos era condição sine qua non para disponibilizar ao público geral. Através de parcerias com a direção da EERP-USP, projetos foram encaminhados à Petrobras e ao BNDES, mas sem sucesso.

A necessidade de organização do arquivo dificultava o início das pesquisas, impossibilitando a solicitação de verbas através de projetos de grande porte junto a órgãos de fomento. Nesse sentido, o Fundo de Cultura e Extensão Universitária da USP foi muito importante para que os trabalhos de conservação preventiva e organização do arquivo recebessem o impulso adequado e o arquivo fosse colocado em condições de pesquisa.

Dessa forma, são mostrados, a seguir, os projetos desenvolvidos através de diferentes facetas do arquivo, nesse percurso. 
O Projeto Revitalização do Centro de Memória da Escola de Enfermagem de Ribeirão Preto - USP, financiado pelo Fundo de Cultura e Extensão Universitária da USP, de julho de 2003 a julho de 2004, teve como responsáveis as profas. doutoras Isabel Amélia Costa Mendes, Margarita Antonia Villar Luis e Toyoko Saeki, e colaboração de Luciana Barizon Luchesi. A renovação do projeto foi aprovada para o período de abril de 2006 a abril 2007. As mesmas docentes receberam a aprovação do projeto Cultura e Memória: ampliando a visibilidade e impacto cultural do Centro de Memória da Escola de Enfermagem de Ribeirão Preto - USP para trabalhos de maio a 2008 a maio de 2009, passando, também, a contar com a colaboração de Mara Elisa Oliva Ferreira, funcionária específica para o acervo, designada em 2006, que agilizou muito o processo de organização do acervo.

Em fins de 2008, com a contratação de Luciana Barizon Luchesi como docente da EERP - USP, a coordenação dos projetos voltados à busca de fomento para o arquivo passou para a profas. doutoras Luciana Barizon Luchesi e Toyoko Saeki. Ambas foram igualmente responsáveis pelo projeto Resgate Histórico e Cidadania: Manutenção do Patrimônio Material e Imaterial da Escola de Enfermagem de Ribeirão Preto - USP, com vigência de março de 2010 a março de 20II. De forma geral, esses projetos autorizavam a compra de material para conservação preventiva e pagamento de bolsistas.

Bolsistas, voluntários e docentes buscaram o desenvolvimento de estratégias para que fossem desenvolvidas várias facetas no Cemeerp. Dentre elas, destacam-se:

- Conservação preventiva: treinamentos em grandes institutos para a realização de higienização, organização e acondicionamento adequados.

- Extensão: abertura do acervo ao público e promoção de eventos voltados para o assunto História da Enfermagem e Museus.

- Pesquisa e ensino: incentivo à pesquisa na disciplina História da Enfermagem, que muito avançou. Hoje, a EERP-USP possui um grupo de pesquisa específico, o Laboratório de Estudos em História da Enfermagem (LAESHE), que tem sido o principal veículo de produção de pesquisa, usando fontes do acervo.

- Financiamento: busca por recursos que subsidiem a compra de material específico para conservação preventiva e manutenção de bolsistas.

Dessa forma, o amplo arquivo do Cemeerp, estimado em mais de 25 mil itens - sendo, em sua maioria, suporte iconográfico - possibilita a revisão da história sob nova perspectiva, olhando para história de vencedores e vencidos.

Além disso, as concepções da História Nova clamam pelo resgate das vozes que foram caladas ou deixaram de ser ouvidas no processo histórico documental, que, em grande parte, relata a história dos vencedores.

\section{A IMPORTÂNCIA DE SE PRESERVAR A MEMÓRIA COLETIVA DA ENFERMAGEM}

O movimento francês da História Nova, na Escola dos Annales, ocorreu no final da década de I920, com grande influência no Brasil na década de 1970 [I9]. O novo modelo buscava dar voz a grupos excluídos da história oficial ou rever a história sob novas perspectivas.

O que se observa na literatura e nos documentos é que ao longo da trajetória da EERP-USP, assim como de muitas outras instituições, os grandes feitos e conquistas são atribuídos, algumas vezes, aos grandes nomes e vultos. Hoje, após pesquisas de História Oral, realizadas pelo Laboratório de Estudos em História da Enfermagem (LAESHE), observou-se que alguns avanços da EERP-USP foram construídos também por anônimos, sem desconsiderar a importância dos grandes vultos, mas começam a emergir pessoas à margem da história oficial, que possuem voz importante na trajetória histórica da EERP-USP.

A valorização da história de uma profissão não deixa de ser importante instrumento de poder, pois alicerça a identidade social e imprime prestígio ao grupo [5].

Nesse sentido, a preservação e o estudo da memória coletiva da Enfermagem vêm ao encontro dos objetivos propostos para o período de 2006-2015 (Década de Recursos Humanos em Saúde), apresentados em 2005, durante a Reunião Regional dos Observatórios de Recursos Humanos em Saúde, que aconteceu em Toronto, no Ganadá. Dentre as resoluções, destaca-se a necessidade de fortalecimento dos profissionais de saúde nas Américas [I6, I8].

O desenvolvimento das pesquisas que evocam a memória coletiva, portanto, colaboram não apenas para o fortalecimento dos profissionais de Enfermagem, mas podem, também, constituir importante instrumento de melhora da autoestima profissional e aumento das taxas de recrutamento nas faculdades de Enfermagem. 


\section{OBJETIVOS}

O projeto Resgate Histórico e Cidadania: Manutenção do Patrimônio Material e Imaterial da Escola de Enfermagem de Ribeirão Preto - USP, aprovado em 2010 pela Pró-Reitoria de Cultura e Extensão Universitária da USP, apresenta os seguintes objetivos:

- Promover a informatização do Centro de Memória da EERP-USP.

- Promover a conservação preventiva do material do acervo.

- Disponibilizar um laboratório prático para os cursos do campus da USP que trabalham na área de arquivos e bibliotecas.

- Promoção de eventos voltados para a História da Enfermagem, procurando despertar vocações para pesquisa na área.

- Propiciar suporte técnico para pesquisadores realizem estudos científicos no acervo.

\section{MATERIAIS E MÉTODOS}

\section{- Informatização do Gemeerp}

Desde 2009, o software Personal Home Library (PHL), que trata da aplicação web voltada para a gestão de coleções e bibliotecas [I2], vem sendo utilizado em parceria com o Núcleo de Apoio Bibliográfico da EERP-USP. O objetivo inicial do Cemeerp foi divulgar a coleção bibliográfica pertencente à prof. Glete de Alcântara. Até o início do presente projeto, foram cadastrados 405 livros, alguns raros e que podem ser pesquisados atra-

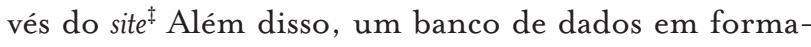
to MySQL, para catalogação de fotografias, mostra-se eficiente, promovendo o acesso rápido à informação e evitando a manipulação desnecessária das fotografias. Até o momento, I3.439 fotografias foram digitalizadas.

- Conservação preventiva do material do acervo

Constantemente, é realizado diagnóstico de identificação do acervo, para higienização e manutenção da

$\ddagger$ http://www2.eerp.usp.br:8080/cgi-bin/wxis.exe?IsisScript=phl. xis\&cipar=phl8.cip\&lang=por. conservação do arquivo, identificando-se, assim, aqueles que apresentam maior risco de deterioração e perda da informação.

Houve aquisição de materiais de proteção, tais como luvas, máscaras, óculos de proteção e aventais para os trabalhos de higienização documental. Os documentos são corretamente armazenados em papel com $\mathrm{pH}$ neutro e folha de poliéster. Além disso, o processo de digitalização do acervo fotográfico, com uso de scanner, promove a redução drástica da manipulação dos originais pelos usuários, garantindo a longevidade dos documentos. Para o material audiovisual é realizado trabalho de rebobinamento e passagem para DVD, uma vez que é importante atualizar o suporte da fonte para sua longevidade, assim como a gravação digital de fitas cassete através de aparelhos de gravação digital. As entrevistas que retratam períodos importantes da história da instituição passaram por processo de transcrição e as famílias ou o(s) entrevistado(s) estão sendo localizados, a fim de solicitar permissão de uso e transferência de direitos autorais [I4].

- Disponibilizar um laboratório prático para os cursos do campus da USP que trabalham na área de arquivos e bibliotecas

O curso de Giências da Informação e Documentação da Faculdade de Filosofia, Giências e Letras de Ribeirão Preto da USP, desde 2006, tem utilizado o espaço do Cemeerp como campo de estágio curricular e extracurricular, fato que muito colabora para o avanço do conhecimento em gestão arquivística no acervo, contando, em alguns momentos, com assessoria de docentes desse curso.

Além disso, o Cemeerp continua a ser espaço de capacitação de profissionais para a realização de conservação preventiva e gestão de arquivo histórico. Durante o tempo de vigência desse projeto, foram realizados cursos nos meses de abril de 20I0, abril, agosto e setembro de 20II e durante a semana de aniversário da EERP-USP, totalizando 64 participantes.

- Promoção de eventos voltados para a História da Enfermagem, procurando despertar vocações para pesquisa na área

O principal evento científico, atualmente organizado pelo Gemeerp em parceria com outros colegiados, é a Semana Professora Glete de Alcântara, que surgiu em 2004 para homenagear a passagem dos trinta anos de falecimento 
da professora Glete de Alcântara ${ }^{\S}$, discutindo a pesquisa em História da Enfermagem. A semana foi idealizada pelos voluntários do Centro de Memória daquele ano, com o apoio da direção da EERP-USP e da Comissão de Cultura e Extensão Universitária desde sua primeira edição. Em 2008, o Departamento de Enfermagem Psiquiátrica e Giências Humanas da EERP-USP uniu-se ao projeto. Desde então, foram sete edições da Semana Professora Glete de Alcântara e quatro Mostras Científicas de História da Enfermagem, atingindo impacto nacional em 2009.

O evento de 2009 foi o ponto crucial para a criação do Laboratório de Estudos em História da Enfermagem (LAESHE), fruto de parceria, desde 2006, entre pesquisadores do Departamento de Enfermagem Psiquiátrica e Ciências Humanas da EERP-USP e Laboratório de Pesquisa em História da Enfermagem (LAPHE), da Escola de Enfermagem Alfredo Pinto, Unirio. Hoje, o LAESHE congrega docentes de ambas as instituições, além de docentes da Escola de Enfermagem de São Paulo e Universidade de Alberta, Canadá, com o total de 9 pesquisadores e quinze pesquisadores em formação. O LAESHE responde, hoje, por mais de $90 \%$ da pesquisa científica realizada em fontes do Cemeerp e, desde 20II, participa da comissão executiva da Semana Professora Glete de Alcântara.

O projeto atual, junto à Pró-Reitoria de Cultura e Extensão, promove várias assessorias técnicas para pesquisadores, desde ajuda na busca de fontes em banco de dados, até orientação e disponibilização de equipamento de proteção para manuseio, se necessário. A disponibilidade da informação digital é feita mediante aprovação em Comitê de Ética pertinente.

No período de vigência do atual projeto, além das ações de catalogação e conservação, houve 357 visitas ao acervo, sendo 55 alunos de ensino médio, 286 de público em geral, dos Estados de São Paulo, Rio de Janeiro e Minas Gerais, e I6 visitantes estrangeiros provenientes dos seguintes países: México, Canadá, Nicarágua, Costa Rica, Guatemala e outros. Foram promovidos eventos culturais, de extensão e científicos que mobilizaram mais de IO4 pessoas.

A atualização do site do Cemeerp, realizada em 20II, também colabora para a divulgação das atividades do acervo".

$\S$ A professora faleceu em 3 de novembro de I974.

If http://www.eerp.usp.br/corporate-historia-centro-memoria-hist-evolucao/.
Estudo realizado em parceria com o LAESHE localizou 98\% das egressas das turmas de I957-I96I, trabalho de dois anos que envolveu muita investigação. Hoje, todos os eventos promovidos pelo Cemeerp ou LAESHE são encaminhados para essas egressas. Nesse momento, as turmas da década de I960 estão sob investigação. Boa parte da produção do LAESHE pode ser acessada gratuitamente por meio do site $^{* *}$.

\section{CONCLUSÕES}

O Cemeerp constitui espaço privilegiado não apenas pelo seu acervo, mas pela potencialidade de agregar formação cultural e científica, lazer, valorização profissional, além de capacitação para estudantes das áreas de Biblioteconomia, História, Moda, Ciências da Informação e Documentação, entre outros. Trata-se, também, de importante recorte da história da Universidade de São Paulo.

A valorização da memória coletiva é primordial no campo da Enfermagem. Espaços como o Centro de Memória possibilitam olhar crítico acerca dos movimentos históricos da construção profissional, ajudando no entendimento do presente, de modo reflexivo, e na elaboração de alternativas possíveis e desejáveis para o avanço da Enfermagem com responsabilidade social e humanismo, valorizando a integralidade do ser humano que é cuidado e que de si mesmo cuida.

\section{REFERÊNCIAS BIBLIOGRÁFICAS}

[I] ALCÂNTARA. G. Memorial. I963. I8 p. Concurso de provimento efetivo do cargo de professor da cadeira no. 4, História da Enfermagem e Ética Aplicada à Enfermagem - Escola de Enfermagem de Ribeirão Preto, Universidade de São Paulo, Ribeirão Preto, r963(a).

[2] _. A enfermagem moderna como categoria profissional: obstáculos à sua expansão na sociedade brasileira. 1963. I28p. Tese de Cátedra - Escola de Enfermagem de Ribeirão Preto, Universidade de São Paulo, Ribeirão Preto, 1963(b).

[3] BRASIL. Lei no. I.467, de 26 de dezembro de I95I. Dispõe sobre a organização e finalidade da Faculdade

\footnotetext{
** http://www2.eerp.usp.br/laeshe.
} 
de Medicina de Ribeirão Preto, da Universidade de São Paulo. Diretoria Geral da Secretaria de Estado dos Negócios do Governo, São Paulo, 27 dez. I95I.

[4] BRASIL. Ministério da Gultura. Patrimônio cultural e Histórico. Disponível em: <http://www.brasil. gov.br/sobre/cultura/patrimonio/patrimonio-material-e-imaterial>. Acesso em: 9 set. $201 \mathrm{I}$.

[5] BARREIRA, I. A. Memória e história para uma nova visão da enfermagem no Brasil. Revista Latino-Americana de Enfermagem, v. 7, n. 3, p. 87-93, I999.

[6] BARREIRA, I. A; BAPTISTA, S. S. La investigación y la documentación en la historia de la enfermería en Brasil. Escola Anna Nery Revista de Enfermagem, v. 4, n. 3, p. 395-403, 2000.

[7] CALDAS, N. P. A experiência da criaçäo do Centro de Memória da Faculdade de Enfermagem da UERJ. Escola Anna Nery Revista de Enfermagem, v.4, n.3, p.347-57, 2000.

[8] CAMPOS, E. S. (org.) História da Universidade de São Paulo, 2. ed. São Paulo: Editora da Universidade de São Paulo, 2004.

[9] CARVALHO, A. C. Escola de Enfermagem da Universidade de São Paulo: resumo histórico - I942I980. Revista da Escola de Enfermagem da USP, v.I4, supl., I980, 27Ip.

[IO] ESCOLA DE ENFERMAGEM DA UNIVERSIDADE FEDERAL DE MINAS GERAIS. Centro de Memória da Escola de Enfermagem. Disponível em: <http://www.enf.ufmg.br/centrodememoria/estrutura.php>. Acesso em: 09 set. $201 \mathrm{II}$.

[II] ESCOLA DE ENFERMAGEM DE RIBEIRÃO PRETO. Aspectos Históricos. Disponível em: <http://www2.eerp.usp.br>. Acesso em: I set. $201 \mathrm{I}$.

[i2] FILHO, E. M. S. O. Personal Home Library. Disponível em: 〈http://www.elysio.com.br >. Acesso em: 9 set. $20 I I$.

[i3] LOMÔNACO, N. V. et al. Centro de Memória da EERP (CEMEERP): Folder institucional. Ribeirão Preto: Escola de Enfermagem de Ribeirão Preto USP, I990.

[I4] LUCHESI, L .B. et al. Redescobrindo o Centro de Memória da Escola de Enfermagem de Ribeirão Preto: relato de experiência. Escola Anna Nery Revista de Enfermagem, v. IO, n. 3, p. 565-7I 2006.

[I5] MENDES, I. A. G. et al. A REBEn no contexto da História da Enfermagem Brasileira: a importância da memória de dra. Glete de Alcântara. Revista Brasileira de Enfermagem, v. 55, n. 3, p. 270-74, 2002.

[i6] MENDES, I. A. G.; MARZIALE, M. H. P. Década de recursos humanos em saúde: 2006-20I5. Revista Latino-Americana de. Enfermagem, v. I4, n. I, p. I-2, 2006. Editorial.

[i7] OGUISSO, T. Memória e história: Centro Histórico-Cultural da Enfermagem Ibero-Americana. Escola Anna Nery Revista de Enfermagem, v. 4, n. 3, p. 359-67, 2000.

[i8] PAN AMERICAN HEALTH ORGANIZATION. Toronto Call to Action for a Decade of Human Resources in Health in the Americas (2006-2015). Regional Meeting of the Observatory of Human Resources in Health, 4-7 October, 2005. Disponível em: <http://www.observarh.org/fulltext/torontocalltoaction.pdf $>$. Acesso em: 9 set. 20 II.

[ig] SAUTHIER, J. Memória e história: O Centro de Documentação da Escola de Enfermagem Anna Nery. Escola Anna Nery Revista de Enfermagem, v. 4, n. 3, p. 339-46, 2000.

[20] SILVAJUNIOR, O. G. et al. Implantação do Arquivo Setorial Enfermeira Maria de Castro Pamphiro. In: IV ENCONTRO DE PROFESSORES E PESQUISADORES DE HISTÓRIA DA ENFERMAGEM DO RIO DE JANEIRO, 2006, Rio de Janeiro. Anais... Rio de Janeiro: UNIRIO, 2006. p. 43-43.

[2I] UNIVERSIDADE DE SÃO PAULO. Escola de Enfermagem de Ribeirão Preto. Ata da $4^{\mathrm{a}}$. Reunião da Comissão de Memória Histórica realizada em setembro de I989. Ribeirão Preto - SP. 